\title{
Factors Associated with Lymphoedema Management Success Among Podoconiosis Patients in Wolaita Zone, Southern Ethiopia
}

\author{
Bereket Alemayehu $^{1, *}$, Mihiretu Alemayehu ${ }^{2}$ \\ ${ }^{1}$ Biomedical Science Division, Department of Biology, College of Natural and Computational Sciences, Wolaita Sodo University, Sodo, \\ Ethiopia \\ ${ }^{2}$ School of Public Health, College of Health Sciences and Medicine, Wolaita Sodo University, Sodo, Ethiopia
}

Email address:

bereketalemayehu@gmail.com (B. Alemayehu),mihiretua@gmail.com (M. Alemayehu)

${ }^{*}$ Corresponding author

To cite this article:

Bereket Alemayehu, Mihiretu Alemayehu. Factors Associated with Lymphoedema Management Success Among Podoconiosis Patients in Wolaita Zone, Southern Ethiopia. Science Journal of Public Health. Vol. 5, No. 5, 2017, pp. 392-400. doi: 10.11648/j.sjph.20170505.16

Received: June 5, 2017; Accepted: June 21, 2017; Published: August 30, 2017

\begin{abstract}
Podoconiosis is non-filarial elephantiasis of lower legs which predominantly affects barefooted people. In later clinical stages, the disease manifests lymphoedema of the lower legs with mossy and nodular changes to the skin. The disease progressively disables patients when they are continuously exposed to irritant soils. The present study aimed to assess factors associated with lymphoedema management success among podoconiosis patients. A community based cross-sectional study was conducted from February to May, 2014 among podoconiosis patients. A two-stage sampling technique was employed to randomly select a total of 845 study subjects from 4 districts of Wolaita zone. To collect data, interviewer administered questionnaire was used along with observation. Data were entered and analyzed by using SPSS version 20. Bivariate and multivariable logistic regressions were used to select the candidate and the predictor variables respectively. Majority (71.2\%) of the study subjects managed their lymphoedema successfully. Three fourth of the study participants were adhered to the stepwise lymphoedema management procedures. Acute dermatolymphangioadenitis (acute attack), was manifested rarely among $61.4 \%$ of the subjects after completion of the treatment. Patients' adherence to stepwise treatment procedures, completion of treatment regimen, duration of follow-up, attitude towards lymphoedema management, age and literacy were found to be the main predictors of lymphoedema management success. The present study assessed main factors associated with lymphoedema management success in podoconiosis. Duration of treatment follow-up, adherence to stepwise treatment procedure, completion of treatment, attitude on disease management and community perceptions towards patients were the main factors associated with successful lymphoedema management. Strict supervision and follow-up of patients to reinforce the home-based lymphoedema care is a vital precondition to successfully manage lymphoedema. Furthermore, longitudinal studies have to be conducted to bring a better lymphoedema management scenario with a higher success rate.
\end{abstract}

Keywords: Podoconiosis, Elephantiasis, Lymphoedema management, Wolaita Zone, Ethiopia

\section{Introduction}

Podoconiosis, endemic non-filarial elephantiasis, is a chronic lymphatic disease caused by long term exposure to irritant volcanic soil which predominantly affects barefooted people [1]. This disease mainly manifests swelling of the lower legs with mossy and nodular changes to the [2]. As a result, it causes a considerable disability among affected people [3]. Eventhough the etiology of the disease is not fully understood, evidences suggest that exposure to irritant soil which contains colloid-sized mineral particles of common clay elements have a significant role to cause the disease among genetically susceptible individuals living in endemic areas $[2,4]$.

The highest prevalence is reported across tropical Africa, where heavy rainfall and seasonal temperature variations are the causes for the breakdown of basaltic soils into particles that penetrate the skin, and provoke 
endolymphangitis and subsequent lymphoedema [5]. Ethiopia is one of the countries with a high disease burden where nearly $18 \%$ of the entire population is exposed to irritant soil. As a result, more than a million people are estimated to be affected by the disease with average prevalence above $5 \%$ in endemic areas $[6,7]$. The country loses as high as 200 million USD per year attributable to this neglected tropical disease [8]. Though less attention is given to podoconiosis, it is more common than the focused diseases such as HIV/AIDS, tuberculosis and filarial elephantiasis in endemic areas of the country [7, 8]. This disease mainly affects people of productive age group. Studies from southern Ethiopia has shown that podoconiosis patients lose forty five percent of their productive work days, costing a single zone more than 16 million USD per year [9].

Podoconiosis progressively increases disability when people are continuously exposed to irritant soils. However, the condition can be reverted with simple and affordable treatment procedure [10]. Initially the disease begins its manifestation by a burning sensation in the foot and lower leg, plantar edema and hyper-keratosis with the formation of moss-like papillomata. Later, the swelling may be either soft and fluid or hard and fibrotic often associated with multiple hard skin nodules $[11,12]$.

A simple affordable treatment approach, which is shared from the management of lymphoedema caused by lymphatic filariasis, has been developed to treat podoconiosis in resource poor settings [13]. The treatment regimen comprises: training about the etiology of podoconiosis and the rationale behind secondary preventative measures such as foot hygiene, involving daily washing with soap, water and dilute bleach (acting as an antiseptic), the regular use of emollient and/or antifungal (e.g. Whitfield's ointment), elevation of the limb at night, and emphasis on the importance of consistent use of shoes and socks. In addition, educational and social support messages on prevention and treatment of the disease are given, and social and spiritual support are offered [14-17]. The success rate of lymphoedema management can be evaluated by assessing the presence or absence of posttreatment outcomes (benefits) [16, 17]. These are: decrement of bad odor of leg, reduction of foot swelling, subsidence of pain, disappearance of entry lesions, reduction in frequency of acute attack, improvement in selfconfidence, being hopeful for his/her future life, and returning back to his/her regular work/school [16]. Therefore, the present study aimed to assess factors associated with lymphoedema management success among podoconiosis patients.

\section{Methods}

\subsection{Study Area and Design}

A community based cross-sectional study was conducted in Wolaita Zone, Southern Ethiopia, from
February to May, 2014 to assess the factors associated with lymphoedema management success among podoconiosis patients. Wolaita zone is one of the 13 zones of Southern Nations, Nationalities and Peoples' Region (SNPPR) located $330 \mathrm{~km}$ south of Addis Ababa, the capital of Ethiopia. The zone has a total population of 1,926,693 in 2016 as projected from 2007 national census. This zone is characterized by high proportion $(88.3 \%)$ of rural population [18] whose livelihood is based on agriculture, in which contact with soil is a routine activity that may predispose them to podoconiosis. In the area, it was reported that around 1.7 million inhabitants are known to bear a heavy podoconiosis burden [6].

The Mossy Foot Treatment and Prevention Association (MFTPA), later named as "Mossy Foot International (MFI)", is a non-governmental organization which was founded in 1998 in Wolaita Zone, Southern Ethiopia. This organization pioneered the treatment and prevention of podoconiosis in Ethiopia as well. In the current study area, MFI has worked a lot to reduce the burden of the disease $[6,19]$. Until recently, the organization has provided preventive, palliative and rehabilitative service for around 35,000 patients annually through the outreach clinic services [19].

\subsection{Study Population and Sampling}

A two stage sampling technique was employed to select study participants. Four districts were randomly selected from the twelve rural districts of the zone in which MFTPA had outreach clinic sites. Then, 845 study participants, who had been the beneficiaries of MFI, were selected by using systematic sampling technique from the list of podoconiosis patients registered at outreach clinic sites.

Sample size was calculated using single population proportion formula with the following assumption. A 50\% proportion of success rate (to obtain maximum sample size), $95 \%$ confidence interval, $5 \%$ precision, and design effect of 2 were used. As a result, the minimum sample size needed for this study was 768 . After adding $10 \%$ non-response rate, the total sample size determined for this study was 845 .

\subsection{Data Collection Procedure}

A pretested interviewer administered questionnaire was used along with observation to collect data. The English version of the questionnaire was translated into the local Wolaita language for interview. Eight well trained nurses were used to fill the questionnaire, which was prepared by including all relevant variables based on the objectives of the study. Each data collector was assigned a guide from MFI-outreach clinic to lead to the houses of study participants. In order to minimize social desirability bias, the data collection was done by non-MFI clinic staffs that were selected from other vicinity. The questionnaire was designed to include questions about socio-demographic characteristics, clinical history, and treatment seeking behavior, shoe wearing habits and personal hygiene practices, treatment duration and outcome, and perception 
of the subjects after treatment. Lymphoedema management success was then determined based on self reported response and observation [17].

\subsection{Data Management and Analysis}

Data was entered into computer and analyzed by using SPSS version 20. Descriptive statistics was used to describe the study population by explanatory variables. Bivariate logistic regression was used to select candidate variables, and the independent predictors of lymphoedema management success were determined using backward stepwise multivariable logistic regression after colinearity test was checked. Odds ratio, along with $95 \%$ confidence interval was used to show the strength of association and all statistical tests were considered significant at alpha $<0.05$.

\subsection{Quality Control}

Intensive training was given to data collectors prior to actual data collection. A pilot test was done in adjacent district which was not included in the main survey to acquire common understanding. Regular supervision was done by supervisors and investigators.

\subsection{Ethical Consideration}

Ethical approval was received from Ethical Review Committee of the College of Natural and Computational Science, Wolaita Sodo University. Permission was obtained from MFI head office and respective outreach clinic sites to access information of patients. Finally, oral consent was obtained from study individuals participated in the interview. Since most of the study populations were not able to read and write, verbal consent was obtained from each study participant. This use of verbal consent was approved by the ethical review committee. Consent was obtained from parents or legal guardians for children aged less than 18 years (the legal age for giving consent for research in Ethiopia). Participants were informed that their responses would be kept confidential and they had full rights to participate or not in the study.

\subsection{Operational Definitions}

(a) Successful lymphoedema management: a self reported and observed alteration in clinical characteristics determined based on the eight post-treatment outcomes specified below. To say the lymphoedema management success was attained, all of eight questions would be answered "Yes" either by the respondent or observed by the data collector.

(b) Unsuccessful lymphoedema management: was determined when at least one of the questions specified below was answered "No".

Variables used to measure lymphoedema management success [16]:

(a) Disappearance of bad odor of the leg (self reported and observed)

(b) Reduction of foot swelling (self reported and observed)

(c) Subsidence of pain (self reported)

(d) Disappearance of entry lesions (observed)

(e) Reduction in frequency of acute attack (self reported)

(f) Improvement in self-confidence (self reported)

(g) Being hopeful for his/her future life (self reported)

(h) Returning back to his/her regular work/school (self reported)

(1) 'Stepwise treatment procedure' was defined as the patients' practice to maintain the hygiene and foot care interventions in following order : 1) washing the foot by using water and soap and then drying, 2) application of antiseptics, 3) elevation of the leg, 4) exercise and 5) regular use of socks and shoes.

(2) 'Completion of treatment regimen' was defined as treatment completion by patient after the full course of the initial treatment given at clinics.

(3) 'Duration of treatment follow-up' was defined as patients' visit to clinics for further treatment, care and support after completion of treatment regimen.

(4) 'Knowledge on management of podoconiosis': was calculated after a number of questions were asked, and correct answers were given score 1 and incorrect answers were given score 0 . The sum was computed and those who scored above the mean were labeled as having 'good' knowledge while their counterparts were labeled as having 'poor' knowledge.

(5) 'Attitude towards podoconiosis management': was measured with lickert scale ranging from 'strongly disagree' to 'strongly agree' and mean score was computed. Participants were categorized as having 'positive' attitude if their score was above the mean score and 'negative' attitude if the score was below the mean score.

\section{Results}

\subsection{Socio-Demographic Characteristics of Respondents}

Eight hundred and twenty patients were involved in the study yielding $97 \%$ response rate. The mean and standard deviation of age of patients were 45.6 and 12.8 years respectively. One third $(32.7 \%)$ of study participants were farmers, followed by housewives and merchants, which accounts for $25.7 \%$ and $18.3 \%$ respectively (Table 1 ). 
Table 1. Socio-demographic characteristics of podoconiosis patients, Wolaita Zone, 2016.

\begin{tabular}{|c|c|c|}
\hline Variable & & Frequency (\%) \\
\hline \multicolumn{3}{|l|}{ Sex } \\
\hline & Male & $380(46.3)$ \\
\hline & Female & $440(53.7)$ \\
\hline \multicolumn{3}{|l|}{ Age } \\
\hline & $<20$ & $40(5.7)$ \\
\hline & $20-34$ & $132(18.8)$ \\
\hline & $\geq 35$ & $532(75.6)$ \\
\hline \multicolumn{3}{|l|}{ Religion } \\
\hline & Protestant & $720(87.8)$ \\
\hline & Orthodox & $60(7.3)$ \\
\hline & Catholic & $40(4.9)$ \\
\hline \multicolumn{3}{|c|}{ Marital status } \\
\hline & Single & $132(16.2)$ \\
\hline & Married & $560(68.6)$ \\
\hline & Divorced & $24(2.9)$ \\
\hline & Widowed & $100(12.3)$ \\
\hline \multicolumn{3}{|c|}{ Educational status } \\
\hline & Cannot read and write & $376(46.1)$ \\
\hline & Read and write & $440(53.9)$ \\
\hline \multicolumn{3}{|c|}{ Occupation } \\
\hline & Farmer & $264(32.7)$ \\
\hline & Housewife & $208(25.7)$ \\
\hline & Potter & $40(5)$ \\
\hline & Weaver & $12(1.5)$ \\
\hline & Merchant & $148(18.3)$ \\
\hline & Daily laborer & $36(4.5)$ \\
\hline & Student & $44(5.4)$ \\
\hline & Unemployed & $32(4)$ \\
\hline & Other & $24(3)$ \\
\hline
\end{tabular}

\subsection{Assessment o Lymphoedema Management Success}

Based on the eight components of lymphoedema management success, disappearance of bad odor $(97.5 \%)$ and reduction of foot swelling $(95.5 \%)$ were the most managed conditions. However, improvement in self-confidence and returning back to regular work/school scored $83.5 \%$ and $84.2 \%$ respectively (Table 2 ).

Table 2. Variables associated with lymphoedema management success among podoconiosis patients, Wolaita Zone, 2016.

\begin{tabular}{llll}
\hline Variables & & Frequency & Percent \\
\hline Bad odor of the leg disappeared & Yes & 788 & $97.5 \%$ \\
& No & 20 & $2.5 \%$ \\
Foot swelling reduced & Yes & 772 & $95.5 \%$ \\
& No & 36 & $4.5 \%$ \\
Pain subsided & Yes & 732 & $90.6 \%$ \\
& No & 76 & $9.4 \%$ \\
Entry lesions disappeared & Yes & 760 & $93.1 \%$ \\
& No & 56 & $6.9 \%$ \\
Frequency of acute attack reduced & Yes & 708 & $91.2 \%$ \\
& No & 68 & $8.8 \%$ \\
Self-confidence improved & Yes & 668 & $83.5 \%$ \\
& No & 132 & $16.5 \%$ \\
Hopeful for his/her future life & Yes & 752 & $94.0 \%$ \\
& No & 48 & $6.0 \%$ \\
Returned back to regular work/school & Yes & 684 & $84.2 \%$ \\
& No & 128 & $15.8 \%$ \\
\hline
\end{tabular}

Overall, $584(71.2 \%)$ of study participants had shown success on all the eight components, whereas $236(28.8 \%)$ hadn't shown success (Figure 1).

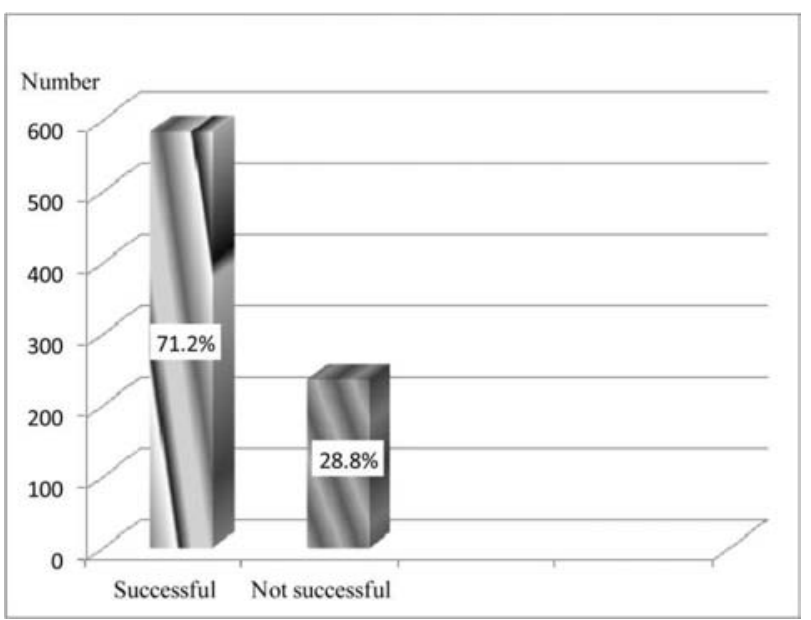

Figure 1. Lymphoedema management success rate among podoconiosis patients, Wolaita Zone, 2016.

\subsection{Patients' Knowledge, Attitude and Practice Towards Lymphoedema Management}

The mean and standard deviation for duration of treatment follow-up was 5.9 and 3.4 years respectively. Majority of them had good knowledge 604(74.8\%) and favorable attitude $612(75.7 \%)$ towards lymphoedema management. Three fourth of the study participants $(74.1 \%)$ responded that they practiced stepwise treatment procedures provided by MFI 
health workers. Only $5.5 \%$ of patients were not satisfied with the overall treatment procedures, whereas the rest $94.5 \%$ were satisfied. Acute attack rarely occurred (i.e. no pain for at least one month) among majority (61.4\%) of patients. However, $3 \%$ and $9.1 \%$ of patients were feeling pain once in a day and once in a week respectively. Even though majority of patients became able to walk as long distance as prepodoconiosis time; still $20.1 \%$ of them could not walk with full comfort or painlessly (Table 3 ).

Table 3. Patients' knowledge, attitude and practice towards lymphoedema management among podoconiosis patients, Wolaita Zone, 2016.

\begin{tabular}{|c|c|c|}
\hline Variable & & Frequency $(\%)$ \\
\hline \multicolumn{3}{|c|}{ knowledge on management of podoconiosis } \\
\hline & Good & $604(74.8 \%)$ \\
\hline & Poor & $204(25.2 \%)$ \\
\hline \multicolumn{3}{|c|}{ Attitude towards podoconiosis management } \\
\hline & Favorable & $612(75.7 \%)$ \\
\hline & Unfavorable & $196(24.3 \%)$ \\
\hline \multicolumn{3}{|c|}{ Stepwise treatment procedure practice } \\
\hline & Yes & $608(74.1 \%)$ \\
\hline & No & $212(25.9 \%)$ \\
\hline \multicolumn{3}{|c|}{ Duration of treatment follow-up } \\
\hline & $<48$ months & $192(23.6 \%)$ \\
\hline & 48-95 months & $440(54.2 \%)$ \\
\hline & 96 months and above & $180(22.2 \%)$ \\
\hline \multicolumn{3}{|c|}{ Satisfaction with entire treatment regimen } \\
\hline & Satisfied & $752(94.5 \%)$ \\
\hline & Not satisfied & $44(5.5 \%)$ \\
\hline \multicolumn{3}{|c|}{ Frequency of acute attack } \\
\hline & Every day & $24(3.0 \%)$ \\
\hline & Once in a week & $72(9.1 \%)$ \\
\hline & Once in a month & $80(10.2 \%)$ \\
\hline & Rarely (less than a month) & $484(61.4 \%)$ \\
\hline & Not at all & $128(16.2 \%)$ \\
\hline \multicolumn{3}{|c|}{ Legs/feet looked normal } \\
\hline & Yes & $668(81.5 \%)$ \\
\hline & No & $152(18.5 \%)$ \\
\hline \multicolumn{3}{|c|}{ Walk as long distance as pre-podoconiosis time } \\
\hline & Yes & $636(79.9 \%)$ \\
\hline & No & $160(20.1 \%)$ \\
\hline \multicolumn{3}{|c|}{ Perceived community perception towards patient changed positively } \\
\hline & Yes & $740(92.5 \%)$ \\
\hline & No & $60(7.5 \%)$ \\
\hline \multicolumn{3}{|c|}{ Perceived stigma and discrimination } \\
\hline & Reduced & $748(92.6 \%)$ \\
\hline & Not reduced & $60(7.4 \%)$ \\
\hline \multicolumn{3}{|c|}{ Visited by government health extension workers } \\
\hline & Yes & $168(20.8)$ \\
\hline & No & $640(79.2)$ \\
\hline \multicolumn{3}{|c|}{ Time it took for improvement } \\
\hline & up to 6 month & $208(26)$ \\
\hline & $7-12$ month & $292(36.5)$ \\
\hline & 13 - 24 month & $176(22)$ \\
\hline & above 24 month & $124(15.5)$ \\
\hline
\end{tabular}

\subsection{Predictors of Successful Lymphoedema Management}

Variables which showed significant and marginally significant association on bivariate analysis were further tested by multivariable regression in order to control confounding effect. As a result, using backward step-wise multivariable logistic regression; age, occupation, educational status, duration of follow-up, adherence to stepwise treatment procedure, completion of treatment, attitude towards podoconiosis management and perceived community perception towards patient were identified as significant predictors of lymphoedema management (Table $4)$. 
Table 4. Predictors of lymphoedema management success among podoconiosis patients, Wolaita Zone, 2016.

\begin{tabular}{|c|c|c|c|c|c|c|c|c|c|}
\hline \multirow{2}{*}{ Variable } & & \multicolumn{2}{|c|}{ Success } & \multirow{2}{*}{ COR } & \multicolumn{2}{|c|}{$95 \%$ CI } & \multirow{2}{*}{ AOR } & \multicolumn{2}{|c|}{$95 \% \mathrm{CI}$} \\
\hline & & Yes & No & & Lower & Upper & & Lower & Upper \\
\hline \multicolumn{10}{|l|}{ Age } \\
\hline & $\leq 35$ & 108 & 64 & & & & & & \\
\hline & $>35$ & 384 & 148 & $1.54 *$ & 1.07 & 2.21 & $4.02 * *$ & 2.03 & 7.96 \\
\hline \multicolumn{10}{|l|}{ Sex } \\
\hline & Male & 300 & 80 & & & & & & \\
\hline & Female & 284 & 156 & $0.49 * *$ & 0.35 & 0.67 & 2.07 & 1.00 & 4.27 \\
\hline \multicolumn{10}{|c|}{ Educational status } \\
\hline & Can't read \& write & 212 & 164 & & & & & & \\
\hline & Read and write & 368 & 72 & $3.95 * *$ & 2.86 & 5.47 & $2.64 * *$ & 1.49 & 4.67 \\
\hline \multicolumn{10}{|c|}{ Occupation } \\
\hline & Farmer & 208 & 56 & & & & & & \\
\hline & Housewife & 108 & 100 & $0.29 * *$ & 0.20 & 0.43 & 0.42 & 0.16 & 1.05 \\
\hline & Potter & 20 & 20 & $0.27 * *$ & 0.14 & 0.54 & $0.16^{*}$ & 0.04 & 0.70 \\
\hline & Merchant & 128 & 20 & 1.72 & 0.99 & 3.01 & 1.05 & 0.40 & 2.74 \\
\hline & Other & 120 & 40 & 0.81 & 0.51 & 1.28 & 1.91 & 0.83 & 4.41 \\
\hline \multicolumn{10}{|c|}{ Adherence to stepwise treatment procedure } \\
\hline & Yes & 496 & 112 & $6.25 * *$ & 4.35 & 9.09 & $3.03 * *$ & 1.72 & 5.56 \\
\hline & No & 88 & 124 & & & & & & \\
\hline \multicolumn{10}{|c|}{ Attitude towards podoconiosis management } \\
\hline & Positive & 532 & 80 & $18.4 * *$ & 12.41 & 27.3 & $5.64 * *$ & 3.18 & 10.0 \\
\hline & Negative & 52 & 144 & & & & & & \\
\hline \multicolumn{10}{|c|}{ Community perception changed positively } \\
\hline & Yes & 564 & 176 & $8.81 * *$ & 4.85 & 16.0 & $3.29 *$ & 1.24 & 8.73 \\
\hline & No & 16 & 44 & & & & & & \\
\hline \multicolumn{10}{|c|}{ Duration of follow-up for treatment } \\
\hline & $\leq 6$ month & 140 & 68 & & & & & & \\
\hline & $7-12$ month & 160 & 132 & $0.59 * *$ & 0.41 & 0.85 & 1.24 & 0.69 & 2.24 \\
\hline & 13-24 month & 164 & 12 & $6.64 * *$ & 3.45 & 12.8 & $4.78^{* *}$ & 1.95 & 11.7 \\
\hline & $>24$ month & 116 & 8 & $7.04 * *$ & 3.25 & 15.3 & $6.14^{* *}$ & 1.78 & 21.2 \\
\hline \multicolumn{10}{|c|}{ Completed treatment regimen } \\
\hline & Yes & 368 & 40 & $8.35 * *$ & 5.71 & 12.2 & $3.61 * *$ & 1.76 & 7.42 \\
\hline & No & 216 & 196 & & & & & & \\
\hline
\end{tabular}

*- significant at $\mathrm{p}<0.05, * *$ - significant at $\mathrm{p}<0$. Abbreviations: COR, Crude Odds Ratio; AOR, Adjusted Odds Ratio; CI, Confidence Interval

Among the socio-demographic characteristics of study participants, age, educational status and occupations were identified as independent predictors of lymphoedema management success (Table 4). As a result, older patients (above 35 years) were 4 times more likely to show success on lymphoedema management, $\mathrm{AOR}=4.02(2.03,7.96)$. Patients who could read and write had a 3 fold higher chance of having a lymphoedema management success as compared to their counterparts (patients who couldn't read and write), $\mathrm{AOR}=2.64(1.49,4.67)$. Compared to farmers, potters had $84 \%$ less chance of showing success, $\mathrm{AOR}=0.16(0.04$, 0.69).

Among treatment related characteristics, adherence to stepwise treatment procedure, completion of treatment regimen and duration of follow-up were independent predictors of lymphoedema management success. As a result, patients who adhered to stepwise treatment procedures were 3 times more likely to show lymphoedema management as compared to those who didn't adhere, AOR $=3.03$ (1.72, 5.56). Patients who had positive attitude towards podoconiosis management were 6 times more likely to show lymphoedema management success as compared to their counterparts, $\mathrm{AOR}=5.64(3.18,9.99)$.

Patients who responded that the community's perception towards them was changed positively after treatment were 3 times more likely to show success in lymphoedema management when compared to patients who responded that the community's perception was not changed, $\mathrm{AOR}=3.29$ $(1.24,8.73)$.

Patients who completed the treatment regimen they had been receiving from MFI were four times more likely to show success in lymphoedema management as compared to patients who didn't complete treatment regimen, $\mathrm{AOR}=3.61$ $(1.76,7.42)$.

Patients with longer duration of follow-up had better success in lymphoedema management. Accordingly, patients who had a follow-up for 13-24 months were five times more likely to show success in lymphoedema management than patients who followed for less than 6 months, $\mathrm{AOR}=4.78$ $(1.95,11.72)$. Similarly, patients who had a follow-up for more than 24 months were six times more likely to show success in lymphoedema management as compared to those who followed for less than 6 months, $\mathrm{AOR}=6.14(1.78$, 21.18).

\section{Discussion}

Lymphoedema is the main manifestation of podoconiosis. Lymphoedema due to podoconiosis occurs because of changes in the lymphatics and lymph nodes of the lower leg 
upon long term exposure to irritant soil micro-particles. The condition is observed among genetically susceptible individuals who walk bare-footed for long period of time [3, 5,11 , and 12]. It is a clinical condition that affects patients physically and psychologically $[6,8]$.

Effectiveness of lymphoedema management in podoconiosis could be affected by several factors of which any one factor or combination of them may impede the management success. Although measuring treatment outcomes of individuals with podoconiosis is a complex phenomenon due to uncontrolled factors, it was attempted by this study to determine the factors associated with lymphoedema management success among people living with podoconiosis based on the patient's self-report to the key variables used in the present study. Accordingly, based on multivariable logistic regression analysis, eight variables were identified as independent predictors of lymphoedema management success. The identified predictor variables were: age, sex, educational status, occupation, stepwise treatment procedure practice, patients' attitude towards disease management, perceived community perception towards treated patients, duration of the treatment follow-up and completion of treatment regimen.

The overall success rate of lymphoedema management among current study participants was $71.2 \%$. Nearly three fourth of the study patients had knowledge and positive attitude towards lymphoedema management. This finding is in line with a study conducted in Odisha state, India, on experience of a community based lymphoedema management program for lymphatic filariasis, which revealed that majority of study participants who had been enrolled in the program had knowledge towards lymphoedema management [20].

Although all genetically susceptible adults are at risk of acquiring podoconiosis, the present study showed patients older than 35 year of age were four times more likely to show success in lymphoedema management, $\mathrm{AOR}=4.02$ (2.03, 7.96). This might be the result of experiences and knowledge acquired in once life, which builds up as the age of a person increases. The role of education in self-management of lymphoedema was also observed that educated patients were three times more likely to show success in lymphoedema management, $\mathrm{AOR}=2.64(1.49,4.67)$ due to the fact that the more the person is educated, the more he/she is aware of disease prevention and treatment. In addition, occupation of patients had significant association with treatment outcome. Accordingly, potters had $84 \%$ lower chance of success in lymphoedema management as compared to farmers, $\mathrm{AOR}=$ $0.16(0.04,0.69)$. The reason might be potters involved in the present study might have less educational status and lower level of awareness on the disease than farmers.

Attitude of patients towards management and practicing stepwise treatment procedures of podoconiosis were observed to be the most important predictors for achieving success in lymphoedema management. In the present study, patients who adhere to stepwise treatment procedures were 3 times more likely to show success in treatment outcome as compared to their counterparts, $\mathrm{AOR}=3.03(1.72,5.56)$. In addition, patients who had positive attitude towards podoconiosis management were six times more likely to show success in treatment outcome of lymphoedema management, $\mathrm{AOR}=5.64(3.18,9.99)$. In one year follow-up study done by Sikorski et al (2014) to see the effectiveness of a simple lymphoedema treatment regimen in podoconiosis management in Wolaita Zone, Southern Ethiopia, it was observed that patients' self commitment was an important condition to see clinical outcomes of lymphoedema treatment in podoconiosis management [17].

Perception of community towards treated patients also had a significant contribution to achieve success in lymphoedema management. In this study, patients who responded as the community perceived positively towards them were 3 times more likely to show success in lymphoedema management, $\mathrm{AOR}=3.29(1.24,8.73)$, as a result of the fact that patients' acceptance by the community and their involvement in various community-based-activities after treatment encouraged the patients to adhere to the lymphoedema management. This might be due to the fact that the quality of life gained and the social value redeemed after lymphoedema management resulted in change in the formerly deep-rooted negative perception of the community towards podoconiosis patients [19]. The social rehabilitation programs included in the podoconiosis prevention and control package in the MFI in southern Ethiopia might also be a reason for improved social value of the treated individuals [2]. Completion of the treatment regimen was also observed to be an important predictor for lymphoedema management success in people living with podoconiosis. In this study, patients who completed the lymphoedema treatment regimen without discontinuation were four times more likely to show success in lymphoedema management as compared to patients who didn't complete the regimen, $\mathrm{AOR}=3.61 \quad(1.76,7.42)$. Because of lack of awareness on lymphoedema management, patients fail to take every-day care for their disease. One of the reasons for discontinuation of lymphoedema treatment was expectation of the patients to see immediate cure after treatment [16]. Even though achieving success in lymphoedema management is largely dependent on the stage of podoconiosis, patients who received treatment for longer duration of follow-up had better treatment outcomes. Patients who had a treatment follow-up at MFI and practiced homebased self-care for over 24 months were six times more likely to show success in lymphoedema management as compared to those who followed for lesser duration (less than 6 months), $\mathrm{AOR}=6.14(1.78,21.18)$. The longer period it takes to treat lymphoedema is because, the condition requires uninterrupted treatment follow-up and life-long care [16]. The longer treatment regimen required for achieving successful management in lymphoedema due to podoconiosis has economic implications. In a community of the present study area, where people live in low socio-economic level, it was reported by many patients that they were unable to afford for the cost of uninterrupted care and treatment $[9,17$, 19]. 


\section{Conclusion and Recommendation}

Even though the majority of podoconiosis patients showed success in managing their lymphoedema, there were some patients who failed to show success manly because of failure to adhere to the treatment procedure and discontinued treatment follow-up. The present study found that age, educational status, occupation, duration of treatment followup, adherence to stepwise treatment procedure, completion of treatment, attitude towards disease management and community perceptions towards patients were observed to be the main factors associated with successful lymphoedema management. Therefore, providing health education and awareness to the patients in particular and the community in general would reduce the failure of lymphoedema management among people living with podoconiosis. Proper practice of stepwise treatment procedure has a significant impact on the outcomes of lymphoedema management. Institutions working on prevention and control of podoconiosis have to share the burden by strictly supervising and following beneficiaries' progresses towards achievement of the goal. Furthermore, researchers have to conduct longitudinal study to bring better lymphoedema management scenario for podoconiosis with a higher success rate.

\section{Strength of the Study}

(a) This is a community based study in which both observational and interview data collection methods were applied to minimize measurement errors occurring during data collection.

(b) Lymphoedema management success was evaluated by assessing all the eight post-treatment outcomes (benefits), which could yield a comprehensive success rate.

\section{Limitation of the Study}

(a) The use of face-to-face interview might have resulted in susceptibility to social desirability bias.

(b) As a cross-sectional study, this study didn't show temporal relation between variables. Rather, it indicated the presence of association between predictor and outcome variables.

\section{Ethics Statement}

The protocol of this study was ethically reviewed and approved by the Ethical Review Committee of College of Natural and Computational Science in Wolaita Sodo University. Permissions were obtained from Wolaita Zone and District Health Departments to conduct the study. Permission was also obtained from MFI head office and respective outreach clinic sites to access information of patients. Oral consent was obtained from study participants. Participants were informed that their responses would be kept confidential and they had full rights to participate or not in the study.

\section{Acknowledgements}

The authors would like to thank Wolaita Sodo University (WSU) for financial and logistic support to conduct the study. We are also thankful to Mossy Foot International for providing valuable information for this study.

\section{References}

[1] Price E. Podoconiosis: Non-filarial Elephantiasis. Oxford: Oxford Medical. 1990.

[2] Davey G. Podoconiosis, non-filarial elephantiasis, and lymphology. Lymphology. 2010; 43(4):168-177.

[3] Price E. The association of endemic elephantiasis of the lower legs in East Africa with soil derived from volcanic rocks. Trans R Soc Trop Med Hyg. 1976; 4:288-295.

[4] Davey G, GebreHanna E, Adeyemo A, Rotimi C, Newport M, and Desta K. Podoconiosis: a tropical model for geneenvironment interactions? Trans R Soc Trop Med Hyg. 2007; 101(1):91-96.

[5] Price EW. The site of lymphatic blockade in endemic (nonfilarial) elephantiasis of the lower legs. J Trop Med Hyg. 1977; 80:230-7.

[6] Destas K, Ashine M, and Davey G. Prevalence of podoconiosis (endemic non-filarial elephantiasis) in Wolaitta, Southern Ethiopia. Trop Doct. 2003; 33:217-20.

[7] Davey G. Podoconiosis: let Ethiopia lead the way. Ethiop $J$ Health Dev. 2008; 22:1-2.

[8] Davey G, and Newport M. Podoconiosis: the most neglected tropical disease? Lancet. 2007; 369:888-9.

[9] Tekola F, Mariam DH, and Davey G: Economic costs of endemic non-filarial elephantiasis in Wolaita Zone, Ethiopia. Trop Med Int Health. 2006; 11:1136-1144.

[10] Mousley E, Deribe K, Tamiru A, and Davey G. The impact of podoconiosis on quality of life in Northern Ethiopia. Health and Quality of Life Outcomes. 2013; 11:122.

[11] Price EW. Endemic elephantiasis: Early signs and symptoms, and control. Ethiopian Medical Journal. 1983; 21:243-53.

[12] Price EW. Pre-elephantiasic stage of endemic nonfilarial elephantiasis of lower legs: "podoconiosis". Tropical Doctor. 1984; 14:115-9.

[13] Addiss DG, and Brady MA. Morbidity management in the Global Programme to Eliminate Lymphatic Filariasis: a review of the scientific literature. Filaria J. 2007; 6:2.

[14] Wilson SF, Guarner J, Valme AL, Louis-Charles J, Jones TL, and Addiss DG. Histopathologic Improvement with Lymphedema Management, Le'ogâne, Haiti. Emerg Inf Dis. 2004; 10: 1938-46.

[15] McPherson T. Impact on the quality of life of lymphoedema patients following introduction of a hygiene and skin care regimen in a Guyanese community endemic for lymphatic filariasis: a preliminary clinical intervention study. Filaria $J$. 2003; 2 : 1 . 
[16] Dreyer G, Addiss D, Dreyer P, and Noroes J. Basic lymphoedema management: treatment and prevention of problems associated with lymphatic filariasis. Int $J$ infect dis. 2004; 8(5):321

[17] Sikorski C, Ashine M, Zeleke Z, and Davey G. Effectiveness of a Simple Lymphoedema Treatment Regimen in Podoconiosis Management in Southern Ethiopia: One Year Follow-Up. PLoS Negl Trop Dis. 2010; 4(11): e902. doi:10.1371/journal.pntd.0000902.

[18] CSA. Summary and statistical report of the 2007 population and housing census, Federal democratic republic of Ethiopia, 2008.
[19] Davey G, and Burridge E. Community-Based Control of a Neglected Tropical Disease: The Mossy Foot Treatment and Prevention Association. PLoS Negl Trop Dis. 2009; 3(5): e424.

[20] Cassidy T, Worrell CM, Little K, Prakash A, Patra I, Rout J, and Fox LM. Experiences of a Community-Based Lymphedema Management Program for Lymphatic Filariasis in Odisha State, India: An Analysis of Focus Group Discussions with Patients, Families, Community Members and Program Volunteers. PLoS Negl Trop Dis. 2016; 10(2): e0004424. doi:10.1371/journal.pntd.0004424. 